\title{
A test of Murdock's D scale technique using an unusual stimulus set
}

\author{
ROBERT SAXE \\ California State University at Northridge, Northridge, California 91324
}

\begin{abstract}
Murdock's D scale technique predicts that when D\%, a measure of stimulus discriminability, is plotted against stimulus position, a U-shaped curve will always result, with end stimuli being more discriminable than middle stimuli. This prediction was tested on a stimulus set in which the middle stimuli were widely separated and the end stimuli were closely spaced. The middle stimuli were found to be the most discriminable. When the data were adjusted for response bias by Doherty's procedure, they still did not fit the prediction derived from Murdock's method.
\end{abstract}

A strong end effect is usually observed in studies in which subjects make absolute judgments of a set of stimuli which vary in magnitude on one physical dimension. In nearly all such studies one of the two end stimuli (the physically greatest or smallest) is more discriminable than any intermediate stimulus. Murdock (1960) developed a method, called the D scale, for predicting a measure of stimulus discriminability from the physical dimensions of the stimuli in a set. The measure of discriminability used in his method is $\mathrm{D} \%$, the percentage of the total number of correct identifications which are made in response to a given stimulus. To predict $\mathrm{D} \%$ using the $\mathrm{D}$ scale method, the physical dimensions of the stimuli are converted to logs, and the sum of the differences of the log of each stimulus from all others in the set is found. The larger this cumulative difference, the greater the predicted discriminability. This cumulative difference is divided by the total of all such cumulative differences in the stimulus set, and multiplied by 100 to give $\mathrm{D} \%$. Murdock's method predicts the results of several absolute judgment experiments quite well. It further predicts that in any stimulus set, no matter what the spacing of the stimuli, the most discriminable stimulus will be one of the two end stimuli, and that discriminability will be strictly increasing from the least discriminable intermediate stimulus (or stimuli, if there is a tie) to either end stimulus.

Bower (1971), in a discussion of Murdock's theory of stimulus distinctiveness, presents a thought experiment which casts doubt upon this prediction:

"Consider an absolute-judgment experiment or PA (paired-associate) learning experiment arranged as

This paper is based on a thesis submitted to the Department of Psychology, California State University at Northridge, in partial fulfillment of the requirements for the MA degree. The author thanks Drs. William B. Knowles, Robert E. Dear, and Frederick A. Muckler for their assistance, and Dr. Bennet B. Murdock, Jr. for his comments on an earlier draft. This paper is sponsored by Bennet B. Murdock, Jr., who takes full editorial responsibility for its contents.

The author is now at the Graduate School of Management, University of California at Los Angeles. follows: Beginning with three widely spaced stimuli, say, tones of 5,45 , and $85 \mathrm{~dB}$ above the threshold, we then add very many new tones that differ by less than a just noticeable difference (JND) from the high and low tones. For example, by adding two low and two high tones, the new set for discrimination might be $3.9,4.0$, $4.1,45,84.9,85.0$, and $85.1 \mathrm{~dB}$. Murdock's PD (percentage-distinctiveness) measure implies that the end stimuli, 3.9 and $85.1 \mathrm{~dB}$, will be the most distinctive and will have the most correct responses, whereas the middle stimulus, $45 \mathrm{~dB}$, will be least distinctive and have the fewest correct responses. One need not run the experiment to know that the prediction must be wrong; in such an experiment the middle stimulus would surely have the most correct responses, because it stands out from the clumps of confusing tones at the high and low ends of the range (p. 183)."

Based on this logic, a stimulus set having the intermediate stimuli much more widely spaced than the end stimuli was constructed to test the predictions of the D scale technique. Within these constraints, the spacing of the stimuli was determined empirically, in pilot research, such that no stimulus would be identified correctly all of the time, and no two stimuli would be completely confused with each other.

\section{METHOD}

\section{Subjects}

Ten subjects judged the stimuli. Nine were undergraduate psychology students at California State University at Northridge who received credit toward their laboratory participation requirement for taking part in the experiment. One was a graduate student in art history.

\footnotetext{
Apparatus

The stimulus set constructed for the experiment consisted of 12 white plastic squares which varied in edge width. When these are ordered by increasing size, the interstimulus differences between Squares 1, 2, 3, and 4, and between Squares 9, 10, 11, and 12 are quite small in comparison to the interstimulus differences between Squares 4, 5, 6, 7, 8, and 9. The dimensions of the stimuli are given in the first column of Table 1.
} 
Table 1

Observed, Adjusted, and Predicted D\% Values

\begin{tabular}{|c|c|c|c|}
\hline \multirow{2}{*}{$\begin{array}{l}\text { Stimulus } \\
\text { Width }(\mathrm{cm})\end{array}$} & \multicolumn{3}{|c|}{$\mathrm{D} \%$ Value } \\
\hline & Observed & Adjusted & Predicted \\
\hline 4.00 & 5.72 & 12.92 & 9.74 \\
\hline 4.15 & 8.08 & 6.49 & 9.12 \\
\hline 4.30 & 6.09 & 4.46 & 8.63 \\
\hline 4.40 & 8.27 & 5.98 & 8.39 \\
\hline 4.90 & 11.88 & 10.39 & 7.66 \\
\hline 5.90 & 11.50 & 12.44 & 7.03 \\
\hline 7.20 & 12.69 & 11.35 & 7.03 \\
\hline 8.40 & 12.06 & 11.49 & 7.55 \\
\hline 9.60 & 7.28 & 5.80 & 8.46 \\
\hline 9.70 & 6.65 & 4.66 & 8.57 \\
\hline 9.85 & 4.91 & 5.15 & 8.78 \\
\hline 10.00 & 4.85 & 8.88 & 9.04 \\
\hline
\end{tabular}

\section{Procedure}

Each subject was shown the 12 stimuli and told their numbers, one at a time in the following random order: $6,9,7,4$, $5,11,2,3,8,12,10,1$. After 72 warmup judgments to familiarize him with the stimuli and the procedures, each subject was given 30 presentations of each stimulus in a random order, with knowledge of results after each judgment. Twelve response categories (the numbers 1 to 12 ) were used. The stimuli were presented one at a time, on a black background.

\section{RESULTS}

The data for all 10 subjects were pooled into a 12 by 12 confusion matrix (Table 2). In this matrix, rows represent the presentations of a stimulus and columns represent the responses given. An element $\mathrm{Aij}$ of the matrix represents the frequency of response $\mathrm{j}$ to stimulus i. The observed $\mathrm{D} \%$ values were calculated from the main diagonal of the confusion matrix, and were compared to the predicted $\mathrm{D} \%$ values derived from the physical dimensions of the stimuli using Murdock's D scale technique. The observed and predicted values are given in columns 2 and 4 of Table 1.

Doherty (1966) pointed out that Murdock's method is sensitive to one type of response bias. The method does not predict $\mathrm{D} \%$ well for subjects who utilize some responses substantially more than others. Doherty presented a method for adjusting the observed frequencies of correct responses to correct for such response bias. The method gives estimates of what the frequency of correct responses to each stimulus would be if each response were used equally often. The D\% values after adjustment by Doherty's procedure are given in Column 3 of Table 1.

The observed data show that the best discriminability occurred in the middle categories, contrary to the prediction of the D scale technique. For the adjusted data, the best discriminability occurred in an end category. However, neither the observed nor the adjusted data showed the U-shaped curve predicted by the technique. A $\chi^{2}$ goodness of fit test rejected the hypothesis that the observed and predicted frequencies of correct responses were statistically equivalent $\left[\chi^{2}(11)\right.$ $=308.5, \mathrm{p}<.001]$. The hypothesis of statistical equivalence of the adjusted and predicted frequencies of correct responses was also rejected $\left[\chi^{2}(11)=297.0\right.$, $\mathrm{p}<.001]$.

\section{DISCUSSION}

The results indicate that a stimulus set for an absolute judgment task can be constructed in which the best discrimination occurs in the middle categories. The middle stimuli in the set (Squares 5, 6, 7, and 8) had interstimulus differences of up to about 10 times the size of the interstimulus differences of the end stimuli, yet the middle stimuli were identified correctly only about twice as often as the end stimuli. This shows the strength of the end effect and indicates why data showing the best discriminability in middle categories are seldom seen.

The findings are inconsistent with the prediction derived from the D scale technique. It seems that this technique is applicable only for certain conventional stimulus spacings. It also appears that the theory behind it, that discriminability is a function of the differences between the log of a stimulus' physical dimension and the logs of the dimensions of all other stimuli in the set, is not complete.

Table 2

Pooled Confusion Matrix for 10 Subjects

\begin{tabular}{|c|c|c|c|c|c|c|c|c|c|c|c|c|c|}
\hline \multirow[b]{2}{*}{ Stimulus } & \multicolumn{12}{|c|}{ Response } & \multirow[b]{2}{*}{ Sum } \\
\hline & 1 & 2 & 3 & 4 & 5 & 6 & 7 & 8 & 9 & 10 & 11 & 12 & \\
\hline 1 & 92 & 130 & 60 & 18 & & & & & & & & & 300 \\
\hline 2 & 20 & 130 & 104 & 43 & 3 & & & & & & & & 300 \\
\hline 3 & 10 & 65 & 98 & 102 & 25 & & & & & & & & 300 \\
\hline 4 & 2 & 24 & 114 & 133 & 26 & 1 & & & & & & & 300 \\
\hline 5 & & & 7 & 89 & 191 & 13 & & & & & & & 300 \\
\hline 6 & & & & 1 & 71 & 185 & 42 & 1 & & & & & 300 \\
\hline 7 & & & & 1 & 3 & 57 & 204 & 35 & & & & & 300 \\
\hline 8 & & & & & & 2 & 61 & 194 & 38 & 5 & & & 300 \\
\hline 9 & & & & & & 1 & 2 & 38 & 117 & 98 & 39 & 5 & 300 \\
\hline 10 & & & & & 1 & & 2 & 8 & 82 & 107 & 66 & 34 & 300 \\
\hline 11 & & & & & & & 2 & 12 & 68 & 103 & 79 & 36 & 300 \\
\hline 12 & & & & & & & & 6 & 46 & 87 & 83 & 78 & 300 \\
\hline Sum & 124 & 349 & 383 & 387 & 320 & 259 & 313 & 294 & 351 & 400 & 267 & 153 & 3600 \\
\hline
\end{tabular}


Two considerations may weaken the conclusions that can be drawn from this experiment. First, the D scale technique was designed for use with unidimensional stimuli. The squares used in this experiment can be considered to be unidimensional stimuli varying in area, or to be two dimensional stimuli in which length and width provide completely redundant information. If the judgment process is based upon use of two dimensions rather than one, the applicability of the test is open to question. However, the predicted $\mathrm{D} \%$ values are the same whether calculated from the areas or from the edge dimensions of the squares.

Second, the stimuli were introduced to the subjects in a random order, rather than in increasing or decreasing order, as is usual in absolute judgment work. This may have weakened the end effect.
The stimulus set used in this study has a spacing seldom seen in absolute judgment work. Thus the data reported may be useful in the validation of other techniques for predicting the discriminability of stimuli from their physical dimensions.

\section{REFERENCES}

Bower, G. H. Adaptation-level coding of stimuli and serial position effects. In M. H. Appley (Ed.), Adaptation-level theory. New York: Academic Press, 1971, Pp. 175-201.

Doherty, M. E. Response bias and Murdock's D scale. Psychological Bulletin, 1966, 66, 289-290.

Murdock, B. B., Jr. The distinctiveness of stimuli. Psychological Review, 1960, 67, 16-31.

(Received for publication October 17, 1974.)

\title{
Selective attention: Noise suppression or signal enhancement?*
}

\author{
CHARLES W. ERIKSEN $\dagger$ and JAMES E. HOFFMAN $\dagger \dagger$ \\ University of Mlinois, Champaign, Illinois 61820
}

\begin{abstract}
RTs were obtained to single letter displays when an indicator, designating in which of four possible positions the letter would appear, preceded the display by intervals of 0-150 msec. Prior information, even by as little as $50 \mathrm{msec}$ as to the letter's position, resulted in reduction in $\mathrm{RT}$. Controls ruled out the possibility that the effect was attributable to changes in fixation, masking, or facilitating effects of a first signal. Instead, the effect appears to reflect characteristics of a central selective attentional mechanism.
\end{abstract}

The present experiment is a follow-up of an unexpected but incidental finding in a previous experiment (Eriksen \& Hoffman, 1973). We have been investigating characteristics of selective attention in the visual field. The basic procedure has employed a circular display of letters with a target letter defined by a black bar indicator. The S's task has been to name or to respond to the target letter as quickly as possible. In addition to manipulating the display variables, a primary manipulation has been to have the indicator precede the letter display by several hundred milliseconds. Reaction time (RT) to the target letter decreases as the indicator precedes the display out to approximately $300 \mathrm{msec}$.

In this previous experiment a single letter display (only the target letter was presented) was used to assess baseline performance when no noise elements were present in the display. As part of the balanced design, a single letter display was preceded by an indicator at

*This research was supported by Public Health Service Research Grant MH-1206 and United States Public Health Service Research Career Program Award K6-MH-22014 to the first author.

$\dagger$ Requests for reprints should be made to Professor Charles W. Eriksen, Psychology Building, University of Illinois, Champaign, Illinois 61820 .

+ Now with the Department of Psychology, University of Delaware, New ark, Delaw are 19711. stimulus onset asynchronies (SOAs) varying from $0-350 \mathrm{msec}$. In this instance, the indicator designated in which of 12 positions in the visual field (all foveal) the target letter would appear. The unanticipated result was the finding that RT to the single letter display decreased as the indicator preceded the display, an effect comparable to that of 12-letter displays.

Now it is not particularly surprising that with multiletter displays, an $\mathrm{S}$ can respond more rapidly to the target letter if he is given information as to where its location will be several hundred milliseconds before the multiletter display appears. But, with a single letter display, no search or location would appear to be necessary since it is the only stimulus present in the visual field. So we are faced with the question of how this reduction in RT is mediated. Prior research has shown that the facilitation in RT in multiletter displays is not mediated via a change in eye fixation nor to a "priming" effect of a first stimulus upon RT to a second stimulus (Bertelson, 1967; Posner \& Boies, 1971).

An effect of attention upon the input side of perceptual processing has been difficult to demonstrate experimentally (Deutsch \& Deutsch, 1967; Reynolds, 1964; and Egeth, 1967). It has been very difficult to rule out, of any selective effects obtained, interpretations 